\title{
BMJ Open Acupuncture combined with medication for opioid use disorder in adults: a protocol for systematic review and meta- analysis
}

\author{
Zhihan Chen (D) , ${ }^{1}$ Rui Wang, ${ }^{1}$ Min Zhang, ${ }^{1}$ Yitong Wang (D) , ${ }^{2}$ Yulan Ren ${ }^{2}$
}

To cite: Chen $Z$, Wang $R$, Zhang M, et al. Acupuncture combined with medication for opioid use disorder in adults: a protocol for systematic review and meta-analysis. BMJ Open 2020;10:e034554. doi:10.1136/ bmjopen-2019-034554

- Prepublication history for this paper is available online. To view these files, please visit the journal online (http://dx.doi. org/10.1136/bmjopen-2019034554).

Received 25 September 2019 Revised 27 March 2020 Accepted 06 May 2020

D) Check for updates

(C) Author(s) (or their employer(s)) 2020. Re-use permitted under CC BY-NC. No commercial re-use. See rights and permissions. Published by BMJ.

${ }^{1}$ School of Acupuncture Moxibustion and Tuina, Chengdu University of Traditional Chinese Medicine, Chengdu, China

${ }^{2}$ School of Chinese Classics,

Chengdu University of Traditional Chinese Medicine, Chengdu, China

Correspondence to Professor Yulan Ren; ryl@cdutcm.edu.cn

\section{ABSTRACT}

Introduction Opioid use disorder (OUD) is a worldwide health problem. Clinical trials indicated that acupuncture combined with medication is effective in OUD, however, there are different conclusions presented by previous trials. This study is designed to evaluate the efficacy and safety of acupuncture combined with medication in OUD. Methods and analysis PubMed, CENTRAL, Embase, Web of Science, CINAHL, PsycINFO, ProQuest Dissertation and Theses, AMED, OpenGrey, Clinicaltrials.gov and who.int/trialsearch will be searched in September 2019 without a language restriction. Randomised controlled trials (RCTs) and quasi-RCTs which included participants with OUD receiving acupuncture therapy combined with medication versus control group will be included in this study. Two reviewers will independently screen studies, extract data, assess risk of bias by the Cochrane risk of bias assessment tool and assess quality of evidence by Grading of Recommendations, Assessment, Development and Evaluation (GRADE) approach. Any disagreements will be arbitrated by the third reviewer. Data synthesis and analysis will be conducted by using RevMan V.5.3. Subgroup analyses, sensitivity analysis, meta-regression and reporting bias assessment will be conducted if necessary and appropriate.

Ethics and dissemination On account of the nature of this systematic review and meta-analysis, ethical approval is not required. The results will be published in a peerreviewed journal.

PROSPERO registration number CRD42019123436.

\section{INTRODUCTION}

Opioid use disorder (OUD) is a problematic pattern of opioid use leading to clinically significant impairment or distress. ${ }^{1}$ The clinical symptoms of OUD include pain, anxiety, depression, insomnia, craving, weight loss, yawning, tearing, sweating, tremor and so on. ${ }^{2-5}$ 'World Drug Report $2019{ }^{6}$ has indicated that an estimated 271 million people had used drugs in the previous year, moreover, the use of opioids has a high prevalence in Africa, Asia, Europe and North America. In 2017, there were an estimated 53.4 million past-year users of opioids globally. ${ }^{7}$ The

\section{Strengths and limitations of this study}

- The most important databases, international general healthcare databases, subject-specific electronic bibliographic databases, citation index database, dissertations and theses database, grey literature database, and clinical trials registry platform will be searched without a language restriction.

- The two previous systematic reviews and metaanalyses assessed the efficacy of acupuncture in opioid use disorder (OUD), rather than acupuncture therapy combined with medication; the third previous study included participants with substance use disorders, and only included studies published in English before November 2014; the fourth studies included participants with OUD treated by auricular acupuncture; in addition, existing trials came to different conclusions in the efficacy of acupuncture therapy combined with medication in OUD, thus, the results of this study could help clinicians to make clinical decision for treating OUD.

- Considering high heterogeneity may be caused by the varieties of acupuncture styles, medication, opioids and duration of opioids use, subgroup analyses will be conducted on these factors.

- The reliability of this study's results will largely depend on the methodological quality of included trials.

global area under opium poppy cultivation increased by more than a third in 2017, while opium production also remains at record level. ${ }^{68}$ The years of 'healthy' life lost through premature death and disability are dominated by drug use disorders from 1990 to 2017, especially from the use of opioids. Previous studies declared that OUD patients have high risks of death and disability, ${ }^{7910}$ and high infection rates of HIV ${ }^{1112}$ and hepatitis $\mathrm{C}$ virus. ${ }^{12} 13$

Treatments for OUD mainly include pharmacotherapy (eg, opioid agonist maintenance treatment), psychosocial therapy, acupuncture therapy and so on. ${ }^{14-17}$ Acupuncture therapy, which is in wide use for a long 
time in some Asian countries, is an important component of traditional Chinese medicine. Previous clinical studies, which explored the efficacy of acupuncture therapies in OUD, had different conclusions. ${ }^{16}{ }^{18-20}$ Thus, we conducted a systematic review and meta-analysis to discuss the efficacy of acupuncture in treating OUD patients, and indicated that electro acupuncture (EA) could improve craving and depression and transcutaneous acupoint electrical stimulation could improve insomnia and anxiety. ${ }^{21}$ However, some previous studies demonstrated that acupuncture therapy combined with medication is effective in improving OUD, ${ }^{22} 23$ and acupuncture therapy combined with medication is more effective than pure acupuncture therapy or pure medication. ${ }^{1822}{ }^{24}$ It is to be noted that the clinic trials have come to different conclusions in the efficacy of acupuncture therapy combined with medication. ${ }^{24-26}$ Some previous meta-analyses and systematic reviews discussed the efficacy of acupuncture therapies in OUD, not acupuncture therapy combined with medication. ${ }^{2127}{ }^{28}$ Grant $e t a l^{29}$ included participants with substance use disorders, namely alcohol, stimulants and opioids substance use, and only included studies published in English before November 2014. Baker and Chang ${ }^{30}$ included participants treated by acupuncture therapy combined with medication, however, it only included studies on auricular acupuncture. Thus, we will conduct a systematic review and meta-analysis to assess the efficacy and safety of acupuncture therapy combined with medication for patients with OUD, hoping to prove a stronger evidence for the efficacy of acupuncture therapy combined with medication in OUD.

\section{METHODS AND ANALYSIS}

This study will be conducted in accordance with the Cochrane Handbook for Systematic Reviews of Interventions $^{31}$ and the Preferred Reporting Items for Systematic Reviews and Meta-Analyses guidelines. ${ }^{32}$

\section{Search strategy}

The 11 databases will be searched from their inception to September 2019, namely PubMed, Cochrane Central Register of Controlled Trials (CENTRAL), Embase, Web of Science, Cumulative Index to Nursing and Allied Health Literature (CINAHL), PsycINFO, ProQuest Dissertation and Theses, Allied and Complementary Medicine Database (AMED), OpenGrey, Clinicaltrials.gov and who.int/trialsearch. The search terms will include subject headings and free terms, such as "acupuncture", "acupressure", "acupoint*", "meridian*”, "opioid-related disorders", "opiate substitution treatment", "opioid*", "heroin*”, "opium*” and so on. Considering the particularity of the 11 databases, we will make special search strategies for each database. Here, table 1 shows the search strategy for CENTRAL. And there will be no language restriction.

\begin{tabular}{|c|c|}
\hline$\#$ & Searches \\
\hline \#1 & MeSH descriptor: [Acupuncture] explode all trees \\
\hline \#2 & $\begin{array}{l}\text { MeSH descriptor: [Acupuncture Therapy] explode all } \\
\text { trees }\end{array}$ \\
\hline \#3 & MeSH descriptor: [Electroacupuncture] explode all trees \\
\hline \#4 & MeSH descriptor: [Acupressure] explode all trees \\
\hline \#5 & $\begin{array}{l}\text { acupunctur }{ }^{\star} \text { or pharmacopuncture or } \\
\text { pharmacoacupuncture or acupotom* or } \\
\text { electroacupunctur }{ }^{\star} \text { or electro-acupunctur }{ }^{\star} \text { or "electro } \\
\text { acupunctur" or acupressure }^{\star} \text { or needl }\end{array}$ \\
\hline \#6 & $\begin{array}{l}\text { point* or acupoint" or meridian* or jingluo or jing-luo or } \\
\text { "jing luo" or "ching lo" }\end{array}$ \\
\hline \#7 & $\# 1$ or \#2 or \#3 or \#4 or \#5 or \#6 \\
\hline \#8 & $\begin{array}{l}\text { MeSH descriptor:(Opioid-Related Disorders)explode all } \\
\text { trees }\end{array}$ \\
\hline \#9 & $\begin{array}{l}\text { MeSH descriptor: [Opiate Substitution Treatment] } \\
\text { explode all trees }\end{array}$ \\
\hline \#10 & MeSH descriptor: [Heroin] explode all trees \\
\hline$\# 11$ & MeSH descriptor: [Morphine] explode all trees \\
\hline$\# 12$ & MeSH descriptor: [Tramadol] explode all trees \\
\hline \#13 & MeSH descriptor: [Methadone] explode all trees \\
\hline \#14 & MeSH descriptor: [Fentanyl] explode all trees \\
\hline \#15 & MeSH descriptor: [Codeine] explode all trees \\
\hline \#16 & MeSH descriptor: [Hydrocodone] explode all trees \\
\hline \#17 & MeSH descriptor: [Buprenorphine] explode all trees \\
\hline \#18 & MeSH descriptor: [Oxycodone] explode all trees \\
\hline \#19 & $\begin{array}{l}\text { opioid or opioids or opium or opiate or heroin or } \\
\text { morphine or morphia or tramadol or methadone or } \\
\text { fentanyl or phentanyl or codeine or methylmorphine or } \\
\text { hydrocodone or buprenorphine or oxycodone }\end{array}$ \\
\hline \#20 & $\begin{array}{l}\text { narcot* or detoxifi }{ }^{\star} \text { or desintoxi }{ }^{\star} \text { or disintoxi }{ }^{\star} \text { or } \\
\text { disintossi }\end{array}$ \\
\hline \#21 & $\begin{array}{l}\# 8 \text { or } \# 9 \text { or } \# 10 \text { or } \# 11 \text { or } \# 12 \text { or } \# 13 \text { or } \# 14 \text { or } \# 15 \text { or } \\
\# 16 \text { or } \# 17 \text { or } \# 18 \text { or } \# 19 \text { or } \# 20\end{array}$ \\
\hline \#22 & \#7 AND \#21 \\
\hline
\end{tabular}

\section{Inclusion criteria}

Types of studies

Clinical randomised controlled trials (RCTs) and quasiRCTs, which were published in any languages, will be included in this study. Ongoing RCTs and quasi-RCTs will also be included. Crossover trials and cluster RCTs will be excluded, in addition, non-RCTs, observational studies, animal experiments, narrative reviews, systematic reviews, meta-analyses and case reports will be excluded.

\section{Types of participants}

Trials included participants with primarily OUD, who meet diagnostic criteria of the Diagnostic and Statistical Manual of Mental Disorder or the International Classification of Diseases or other validated criteria or clinical assessment will be included in this study. Participants under 18 years of age and pregnant women will be excluded on account of actual clinical conditions. ${ }^{33} 34$ 
There will be no restrictions on participants' gender and race.

\section{Types of interventions}

All types of acupuncture therapies combined with medication will be included in this study. Studies must include at least one of the following comparators:

1. Acupuncture therapies combined with medication versus sham acupuncture combined with medicine.

2. Acupuncture therapies combined with medication versus pure acupuncture therapy.

3. Acupuncture therapies combined with medication versus pure medication treatment.

If there are uncertain interventions, this study will be excluded.

\section{Outcome assessments}

For assessing instant effect and treatment stability, studies which measured outcomes after treatment or in follow-up ( 8 weeks, 12 weeks or longer) will be included.

\section{Primary outcomes}

1. Intensity of withdrawal syndrome.

2. Number of positive urine samples for opioids.

\section{Secondary outcomes}

1. Intensity of pain, anxiety, depression, insomnia and other associated symptoms.

2. Number of participants with relapse.

3. Retention of treatment.
4. Nature and rate of adverse effect.

\section{Data collection and analysis}

Selection of studies

EndNote V.X9 (Clarivate Analytics, Pennsylvania, USA) will be used to manage the search results from above-mentioned databases. In the first step, two reviewers (ZC and RW) will independently identify articles by their title and abstract according to inclusion criteria, and ineligible studies will be removed to trash in EndNote V.X9; in the second step, the two reviewers will independently screen articles by their fulltext version. The two reviewers will cross-check the selection results. Any disagreements will be arbitrated by the third reviewer (YR). The process of selection will be showed in figure 1. Each unique study ID will be allocated to each eligible study, which will comprise last name of first author and year of publication (eg, Chen 2019).

\section{Data extractions}

The two reviewers (ZC and RW) will independently extract the data into self-designed data extraction form. The data extraction form will include basic information (article title, authors, publication data, country), study design (study type, sample size, characteristics of participants, time of drug abuse, daily opioids use, intervention details, duration, outcome measures) and conclusions. The completed data extraction forms will be cross-checked by the two reviewers. Any diversities will be resolved by the third reviewer (YR).

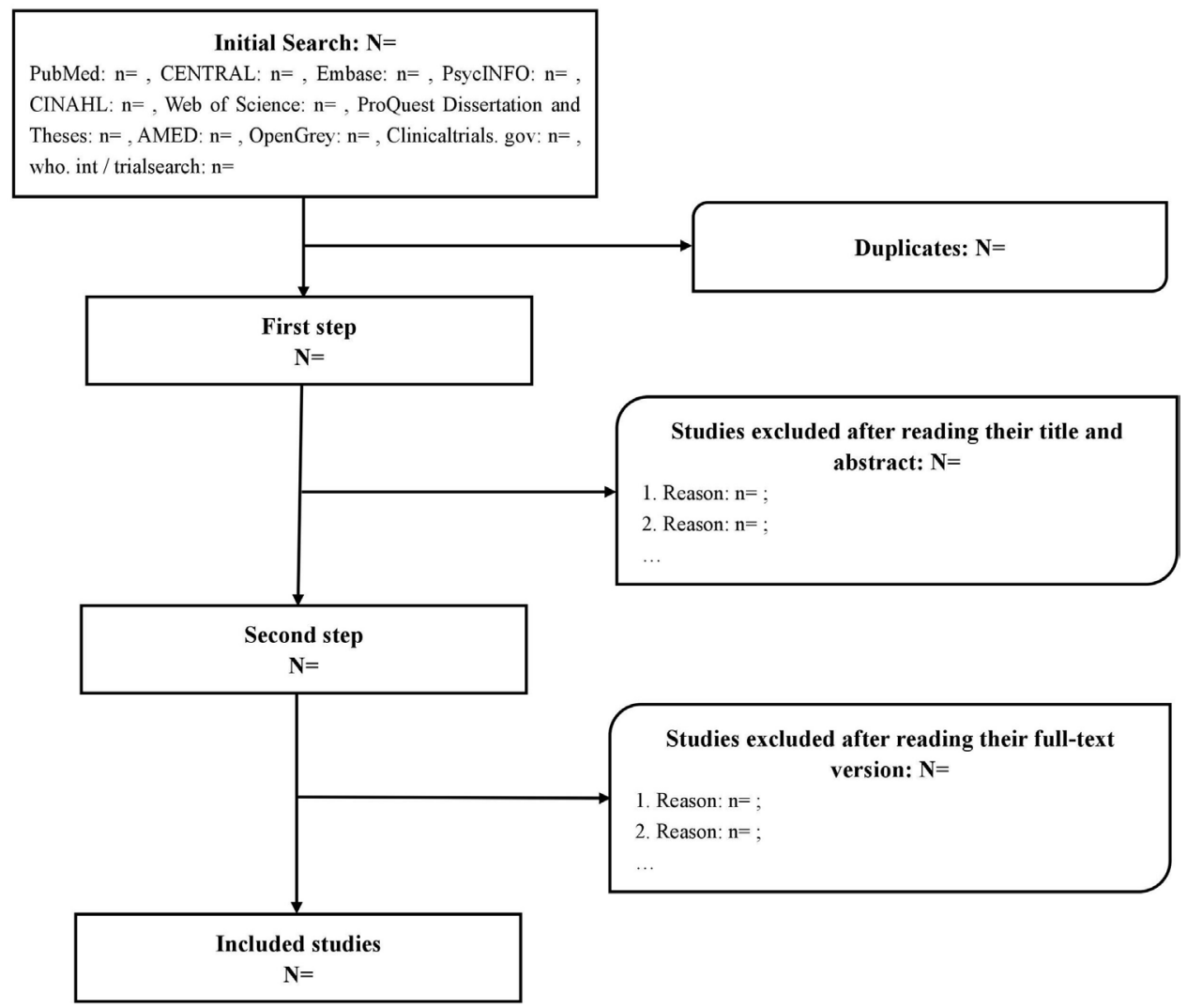

Figure 1 Flow chart of selection. 


\section{Assessment of risk of bias}

The two reviewers (ZC and RW) will assess the risk of bias for each included study by the Cochrane risk of bias assessment tool. ${ }^{31}$ The assessment of risk of bias will include six domains: selection bias (random sequence generation, allocation concealment), performance bias (blinding of participants and personnel), detection bias (blinding of outcome assessment), attrition bias (incomplete outcome data), reporting bias (selective reporting) and other bias. The risk of bias will be graded as low risk, or high risk, or unclear risk. If there is any controversy, the third reviewer (YR) will be consulted. If all key domains are graded as low risk, this study will be graded as low risk; if one or more key domains are graded as high risk, this study will be graded as high risk; if one or more key domains are graded as unclear risk, this study will be graded as unclear risk. ${ }^{31}$ The assessment results will be cross-checked by the two reviewers and the disagreements will be handled by the third reviewer (YR).

\section{Assessment of quality of evidence}

The two reviewers ( $\mathrm{ZC}$ and $\mathrm{RW}$ ) will assess the quality of evidence for outcomes by Grading of Recommendations, Assessment, Development and Evaluation (GRADE) approach. $^{31} 35$ The evidence of included randomised study will be graded as high-quality evidence, or be downgraded to moderate, low or very low quality evidence. The quality of evidence will be assessed by the aspects of study design and implementation, directness of evidence, unexplained heterogeneity, imprecision of results and publication bias. The results of assessment will be cross-checked by the two reviewers and be generated by GRADEprofiler (GRADEpro) V.3.6.1 (Evidence Prime, Ontario, Canada). Any controversy will be handled by the the third reviewer (YR).

\section{Data synthesis and analysis}

Efficacy data from included studies will be analysed by RevMan V.5.3 (The Nordic Cochrane Centre, The Cochrane Collaboration, Copenhagen). For dichotomous outcomes, data will be analysed by risk ratios (RRs) with $95 \%$ CIs. For continuous outcomes, if outcomes are measured by different assessment tools, data will be analysed by standardised mean differences with 95\% CIs; if outcomes are measured by same assessment tools, data will be analysed by mean differences with $95 \%$ CIs.

If there is missing data, we will attempt to contact authors of included study to ask for relevant information. If data are 'missing at random', available data will be analysed; if data are 'not missing at random', we will attempt to contact authors, investigators or contacts recorded in the clinical trials registry platform to obtain data. Nevertheless, if there is no reply, we will impute the missing data with replacement values, and treating these as if they were observed. ${ }^{31}$ Furthermore, sensitivity analysis will be conducted to assess how sensitive results are to reasonable changes in the assumptions that are made, if possible.
Heterogeneity will be assessed by $\mathrm{I}^{2}$. If $\mathrm{I}^{2}$ ranges from $0 \%$ to $40 \%$, the heterogeneity might not be important; if $\mathrm{I}^{2}$ ranges from $30 \%$ to $60 \%$, the heterogeneity may be moderate; if $\mathrm{I}^{2}$ ranges from $50 \%$ to $90 \%$, the heterogeneity may be substantial; if $\mathrm{I}^{2}$ ranges from $75 \%$ to $100 \%$, the heterogeneity may be considerable. ${ }^{31}$ Thus, if $\mathrm{I}^{2}$ is greater than $50 \%$, data will be analysed by using randomeffects model; if $\mathrm{I}^{2}$ is equal to or less than $50 \%$, data will be analysed by using fixed-effect model. In addition, subgroup analyses or meta-regression will be used to explore heterogeneity, and we will try to explain heterogeneity from clinical and methodological differences.

If there is multiple-intervention study, the formulae in the Cochrane Handbook for Systematic Reviews of Interventions will be used to combine relevant intervention groups into a single group. ${ }^{31}$

Due to the varieties of acupuncture styles, medication, opioids, duration of opioids use, and their influence on clinical therapeutic efficacy, subgroup analyses on these factors will be conducted if data are sufficient.

Sensitivity analysis will be conducted to test the robustness of findings. If studies have significant differences in assessment results of risk of bias, we will exclude study with high risk of bias from analysis.

Meta-regression will be conducted to explore heterogeneity by STATA V.14 (StataCorp LLC, Texas, USA), according to time of drug abuse, types of opioids, daily opioids consumption, acupuncture types, therapeutic medicine types and risk of bias (selection bias, performance bias, detection bias, attrition bias, reporting bias and other bias). It is worth noting that meta-regression will be conducted when there is at least 10 studies in a meta-analysis. $^{31}$

Reporting bias include publication bias, time lag bias, duplicate publication bias, outcome reporting bias and so on. For avoiding reporting bias as much as possible, we will search the most important databases, international general healthcare databases, subject-specific electronic bibliographic databases, citation index database, dissertations and theses database, grey literature database and clinical trials registry platform. Moreover, funnel plot will be used to assess reporting bias, if number of included studies exceeds $10 .^{31}$

\section{Ethics and dissemination}

On account of the nature of systematic review and metaanalysis, ethical approval is not required. The results of this study will be disseminated through a peer-reviewed journal.

Authors' contributions ZC proposed this protocol and drafted the manuscript. ZC, RW, MZ, YW and YR participated in revision of the study design. ZC proposed search strategy, and RW and YR revised it. YR revised this manuscript. All authors read and approved the manuscript.

Funding This study was supported by Science \& Technology Department of Sichuan Province (grant number: 2018SZ0071).

Competing interests None declared.

Patient consent for publication Not required. 
Provenance and peer review Not commissioned; externally peer reviewed.

Open access This is an open access article distributed in accordance with the Creative Commons Attribution Non Commercial (CC BY-NC 4.0) license, which permits others to distribute, remix, adapt, build upon this work non-commercially, and license their derivative works on different terms, provided the original work is properly cited, appropriate credit is given, any changes made indicated, and the use is non-commercial. See: http://creativecommons.org/licenses/by-nc/4.0/.

\section{ORCID iDs}

Zhihan Chen http://orcid.org/0000-0002-0237-7501

Yitong Wang http://orcid.org/0000-0002-4968-4410

\section{REFERENCES}

1 American Psychiatric Association. Diagnostic and statistical manual of mental disorders. Fifth Edition. Arlington: VA, American Psychiatric Association, 2013.

2 Wesson DR, Ling W. The clinical opiate withdrawal scale (cows). J Psychoactive Drugs 2003:35:253-9.

3 Meade CS, Lukas SE, McDonald LJ, et al. A randomized trial of transcutaneous electric acupoint stimulation as adjunctive treatment for opioid detoxification. J Subst Abuse Treat 2010;38:12-21.

4 Moeini M, Omidi A, Sehat M, et al. The effects of oxytocin on withdrawal, craving and stress response in Heroin-Dependent patients: a randomized, double-blind clinical trial. Eur Addict Res 2019;25:41-7.

5 Haight BR, Learned SM, Laffont CM, et al. Efficacy and safety of a monthly buprenorphine depot injection for opioid use disorder: a multicentre, randomised, double-blind, placebo-controlled, phase 3 trial. Lancet 2019;393:778-90.

6 United Nations Office on Drugs and Crime. World drug report 2019: Boollet 1. United Nations publication, 2019.

7 United Nations Office on Drugs and Crime. World drug report 2019: Boollet 2. United Nations publication, 2019.

8 United Nations Office on Drugs and Crime. World drug report 2018: Boollet 3. United Nations publication, 2018.

9 Kariisa M, Scholl L, Wilson N, et al. Drug Overdose Deaths Involving Cocaine and Psychostimulants with Abuse Potential - United States, 2003-2017. MMWR Morb Mortal Wkly Rep 2019;68:388-95.

10 Rogers AH, Bakhshaie J, Zvolensky MJ, et al. Pain anxiety as a mechanism linking pain severity and opioid misuse and disability among individuals with chronic pain. J Addict Med 2019.

11 Mathers BM, Degenhardt L, Phillips B, et al. Global epidemiology of injecting drug use and HIV among people who inject drugs: a systematic review. Lancet 2008;372:1733-45.

12 Heimer R, Hawk K, Vermund SH. Prevalent misconceptions about opioid use disorders in the United States produce failed policy and public health responses. Clin Infect Dis 2019;69:546-51.

13 Degenhardt L, Peacock A, Colledge S, et al. Global prevalence of injecting drug use and sociodemographic characteristics and prevalence of HIV, HBV, and HCV in people who inject drugs: a multistage systematic review. Lancet Glob Health 2017;5:e1192-207.

14 Dematteis M, Auriacombe M, D'Agnone O, et al. Recommendations for buprenorphine and methadone therapy in opioid use disorder: a European consensus. Expert Opin Pharmacother 2017;18:1987-99.

15 World Health Organization. Guidelines for the Psychosocially assisted pharmacological treatment of opioid dependence. WHO press, 2009.
16 Bearn J, Swami A, Stewart D, et al. Auricular acupuncture as an adjunct to opiate detoxification treatment: effects on withdrawal symptoms. J Subst Abuse Treat 2009;36:345-9.

17 Dugosh K, Abraham A, Seymour B, et al. A systematic review on the use of psychosocial interventions in conjunction with medications for the treatment of opioid addiction. J Addict Med 2016;10:93-103.

$18 \mathrm{Hu}$ J, Xin Y, Zong L, et al. Clinical research on detoxification with acupuncture. J acupunct tuina sci 2003;1:33-6.

19 Mu J-ping, Liu L, Cheng J-ming, et al. Clinical study on electroacupuncture for post-withdrawal anxiety-depression mood in heroin addicts. J. Acupunct. Tuina. Sci. 2009;7:203-6.

20 Wen T-Q, Yang Z-J, Lei X-L, et al. [Clinical application of acupuncture for treatment of heroin withdrawal syndrome]. Zhongguo Zhen Jiu 2005;25:449-53.

21 Chen Z, Wang Y, Wang R, et al. Efficacy of acupuncture for treating opioid use disorder in adults: a systematic review and meta-analysis. Evid Based Complement Alternat Med 2018;2018:1-15.

22 Zong L, Hu J, Li Y, et al. Comparison of the breaking effects of acupuncture, Chinese medicine and their combination on heroin addiction. Shanghai J Acupuncture Moxibustion 2001;20:1-3.

23 Zeng X, Lei L, Lu Y, et al. Treatment of heroinism with acupuncture at points of the Du channel. J Tradit Chin Med 2005;25:166-70.

24 Chan Y-Y, Lo W-Y, Li T-C, et al. Clinical efficacy of acupuncture as an adjunct to methadone treatment services for heroin addicts: a randomized controlled trial. Am J Chin Med 2014;42:569-86.

25 Zhang PG, Kang B, Zhong Q. Studies on the synergia effect of electroacupuncture and methadone on heroin withdrawal symptoms. Shanghai journal of acupuncture and moxibustion 2004;23:5-6.

26 Rong J, Liu ZY A. Clinical research of scalp acupuncture combined with medical therapy for improving heroin deaddiction's withdrawal symptoms. Zhejiang Journal of Integrated Traditional Chinese and Western Medicine 2016;16:335-7.

27 Jordan JB. Acupuncture treatment for opiate addiction: a systematic review. J Subst Abuse Treat 2006;30:309-14.

28 Boyuan Z, Yang C, Ke C, et al. Efficacy of acupuncture for psychological symptoms associated with opioid addiction: a systematic review and meta-analysis. Evid Based Complement Alternat Med 2014;2014:1-13.

29 Grant S, Kandrack R, Motala A, et al. Acupuncture for substance use disorders: a systematic review and meta-analysis. Drug Alcohol Depend 2016;163:1-15.

30 Baker TE, Chang G. The use of auricular acupuncture in opioid use disorder: a systematic literature review. Am J Addict 2016;25:592-602.

31 Higgins JPT, Green S. Cochrane Handbook for Systematic Reviews of Interventions Version 5.1.0 [updated March 2011]. The Cochrane Collaboration, 2011. Available: www.handbook.cochrane.org

32 Liberati A, Altman DG, Tetzlaff J, et al. The PRISMA statement for reporting systematic reviews and meta-analyses of studies that evaluate healthcare interventions: explanation and elaboration. BMJ 2009;339:b2700.

33 Pani PP, Vacca R, Trogu E, et al. Pharmacological treatment for depression during opioid agonist treatment for opioid dependence. Cochrane Database Syst Rev 2010;9:CD008373.

34 Amato L, Minozzi S, Davoli M, et al. Psychosocial combined with agonist maintenance treatments versus agonist maintenance treatments alone for treatment of opioid dependence. Cochrane Database Syst Rev 2011;10:CD004147.

35 Schünemann H, Brożek J, Guyatt G, et al. Grade Handbook, 2013. Available: http://gdt.guidelinedevelopment.org/app/handbook/ handbook.html 